\title{
Chondrogenic differentiation of bone marrow-derived stem cells cultured in the supernatant of elastic cartilage cells
}

\author{
XIAODIE ZHANG, KE XUE, JIA ZHOU, PENG XU, HUIZHEN HUANG and KAI LIU \\ Shanghai Key Laboratory of Tissue Engineering, Department of Plastic and Reconstructive Surgery, \\ Shanghai 9th People's Hospital, Shanghai Jiao Tong University School of Medicine, Shanghai 200011, P.R. China
}

Received August 17, 2014; Accepted April 30, 2015

DOI: $10.3892 / \mathrm{mmr} .2015 .4113$

\begin{abstract}
Repair of cartilage defects remains a challenge for surgeons, owing to its poor self-repairing capacity. Cartilage tissue engineering, particularly marrow stem cell-based cartilage regeneration, provides a promising option for the regeneration of damaged cartilage. Although producing tissue-engineered cartilage from marrow stem cells appeared to be a feasible method, constructing certain sub-types of cartilage, including elastic cartilage, remains difficult. Therefore, the present study explored the feasibility of constructing elastic cartilage by culturing bone marrow-derived stem cells (BMSCs) in the supernatant of elastic cartilage cells to generate elastic cartilage. The elastic cartilage cells were obtained from the auricle cartilage of a newborn pig, and BMSCs were isolated from pig bone marrow aspirate. The supernatant of the chondrocytes was collected and then used to the culture BMSCs. At various time-points, the differentiation of BMSCs was evaluated by gross view, histological examination and quantitative polymerase chain reaction. BMSCs changed from spindle-shaped cells into polygonal cells with increasing culture time. The expression of collagen II and elastin was observed in the cells cultured in the supernatant of elastic chondrocytes, while no expression was observed in the control cells. Furthermore, the expression of collagen I and collagen X was downregulated in the cells cultured in the supernatant of elastic cartilage cells. The supernatant of elastic cartilage cells promoted the differentiation of BMSCs into elastic cartilage cells, which may be a promising method for constructing certain sub-types of tissue-engineered cartilage.
\end{abstract}

Correspondence to: Dr Kai Liu, Shanghai Key Laboratory of Tissue Engineering, Department of Plastic and Reconstructive Surgery, Shanghai 9th People's Hospital, Shanghai Jiao Tong University School of Medicine, 639 Zhizaoju Road, Shanghai 200011, P.R. China E-mail: drkailiu@163.com

Key words: chondrocytes, elastic cartilage, bone marrow-derived stem cells, tissue engineering, differentiation

\section{Introduction}

Congenital or acquired deformities of the external ear have significant negative effects on patients (1). The cartilage of the external ear, classified as elastic cartilage due to the high abundance of elastic fibers, is known to be avascular, aneural and alymphatic, and therefore, it is difficult for the external ear to be restored following being damaged (2). At present, the most commonly used treatment for deformities of the external ear is an ear-shaped and hand-carved autologous costal cartilage graft which $(3,4)$. Despite the high biocompatibility and immune privilege of this method, it is associated with certain limitations and degrees of success. During this procedure, it is rather difficult to sculpt an anatomic-fidelity auricle shape, possibly resulting in a distorted shape. Furthermore, such method highly depends on the skill and experience of the surgeon $(5,6)$. All of these make it difficult to achieve a satisfying cosmetic result and a shape with long-term stability.

In order to avoid these problems, cell-based tissue engineering has motivated numerous researchers to explore a cure for the deformity (7). Considering the source of cells for tissue-engineered elastic cartilage, autologous chondrocytes are a sensible choice of cell source due to their fast proliferation and being established enough in the generation of auricle cartilage (8). However, it has been reported that chondroctyes rapidly lose their cobblestone-like appearance and function under monolayer culture conditions in vitro, leading to the generation of compromised cartilage tissue (9).

Another candidate cell source are bone marrow-derived stem cells (BMSCs), characterized by self-renewal, a rapid proliferative capacity and the potency of differentiating into chondrocytes (10). At present, numerous methods are known to induce chondrogenic differentiation of BMSCs. The application of transforming growth factor- $\beta$ (TGF- $\beta$ ) and dexamethasone is a classical method. However, at the same time, incubation with TGF- $\beta$ leads to the upregulation of the expression of certain genes, including collagen $\mathrm{X}$, alkaline phosphatase and matrix metalloproteinase 13, which indicates hypotrophic progress, inevitably resulting in calcification and vascularization of the generated cartilage, which is a major drawback besides the high cost (11-13).

Co-culture of auricle chondrocytes and BMSCs may represent a promising method to overcome those hurdles. It has been reported that the production of extracellular matrix (ECM) in 
co-culture occurs earlier than that in chondrocytes or induced BMSCs alone (14). For the co-culture system, it has been reported that BMSCs, either through direct or indirect contact with chondrocytes, are driven to differentiate towards chondrocytes and produce ECM-assembled cartilage $(15,16)$. The underlying mechanism of this inductive effect of chondrocytes on BMSCs is likely to be the secretion of paracrine by the chondrocytes (17). In addition, Hwang et al (18) demonstrated that the supernatant of chondrocytes induced the chondrogenic differentiation of stem cells. To date, it is has not been reported that the supernatant of auricle chondrocytes of piglets drives BMSCs to differentiate towards elastic chondrocytes and produce elastic cartilage.

The present study utilized the supernatant of auricle chondrocytes to drive BMSCs to differentiate towards elastic chondrocytes, and then subjected them to histological and polymerase chain reaction (PCR) analyses. The feasibility of fabricating elastic cartilage through this method was examined and a possible protocol for structuring the desired type of cartilage was provided.

\section{Materials and methods}

Isolation and culture of BMSCs and auricle chondrocytes. All experimental protocols involving animal tissues and cells were approved by the Ethics Committee of Shanghai Jiao Tong University School of Medicine (Shanghai, China).

Bone marrow was obtained from the femur of newborn piglets as described previously (19). Changfeng hybrid piglets (age, 4-7 days; weight, $2 \mathrm{~kg}$ ) were purchased from Shanghai Chuansha Breeding Factory (Shanghai, China). The bone marrow sample was then seeded into culture dishes for the whole-blood adherent method (20), and complete low-glucose Dulbecco's modified Eagle's medium (L-DMEM; Gibco-BRL, Invitrogen Life Technologies, Carlsbad, CA, USA) was added. The complete L-DMEM contained $10 \%$ (v/v) fetal bovine serum (FBS; Gibco-BRL). The cells were incubated at $37^{\circ} \mathrm{C}$ in a humidified atmosphere containing $5 \% \mathrm{CO}_{2}$.

Auricle chondrocytes were harvested from the external auricle of the newborn piglet, which was sacrificed via the aeroembolism method (21). The sample was diced into 1x1-mm pieces, which were submerged in chloromycetin (Sinopharm Chemical Reagent Co., Ltd., Shanghai, China) for 1 hour, followed by digestion with $0.25 \%$ trypsin (Gibco-BRL). Chondrocytes were isolated from the surrounding matrix by collagenase II digestion (Sigma-Aldrich, St. Louis, MO, USA) for $30 \mathrm{~min}$ at room temperature. The cells were seeded at 5,000 cells $/ \mathrm{cm}^{2}$ and expanded in complete H-DMEM (Gibco-BRL).

Flow cytometric analysis. The BMSCs at primary passage were digested, counted and suspended at a density of $1-2.5 \times 10^{6}$ cells $/ \mathrm{ml}$. Subsequently, $200-\mu 1$ aliquots of the cell suspension were transferred into $1.5-\mathrm{ml}$ centrifuge tubes, and the cells were incubated with $1 \mu \mathrm{l}$ anti-rat CD29, anti-human CD34, anti-human CD45 or anti-human CD90 (all BD Biosciences, Franklin Lakes, NJ, USA), respectively, at $4^{\circ} \mathrm{C}$ for $30 \mathrm{~min}$, during which the tubes were gently agitated every $10 \mathrm{~min}$. The samples were then washed three times with phosphate-buffered saline containing 4\% (v/v) FBS. After that, the samples were assessed by flow cytometry (Beckman Coulter FC 500; Beckman Coulter, Inc., Brea, CA, USA).
Table I. Primer sequences for reverse transcription quantitative polymerase chain reaction.

\begin{tabular}{lll}
\hline Gene & \multicolumn{1}{c}{ Primer } & $\begin{array}{c}\text { Product } \\
(\mathrm{bp})\end{array}$ \\
\hline COL1A2 & F: 5'-GGTTTCGGCAAAGTTGGAGG-3' & 218 \\
& R: 5'-GCCCTTTCTTGCAGTTGCC-3' & \\
COL2A1 & F: 5'-CGAGACAGGTGCTGCAAGTC-3' & 141 \\
& R: 5'-TGATCACCTGGTTTCCCACC-3' & \\
COL10A1 & F: 5'-AAAGGTCATGCTGGAGAGCC-3' & 239 \\
& R: 5'-TCATAGTGCTGTTGCCCGTT-3' & \\
Elastin & F: 5'-GGAAAGTACCAGGTGTGGGG-3' & 338 \\
& R: 5'-CCCTGTCCCTGTTGGGTAAC-3' & \\
GAPDH & F: 5'-CCTCAACGACCACTTCGTCA-3' & 237 \\
& R: 5'-GGGTCTGGGATGGAAACTGG-3' &
\end{tabular}

F, forward; R, reverse; COL, collagen.

Chondrogenic differentiation. Auricle chondrocytes at passage 2 were incubated in H-DMEM for $48 \mathrm{~h}$, and the supernatant of the cells was harvested. Following this, the supernatant was centrifuged at $660 \mathrm{xg}$ for $10 \mathrm{~min}$ (17). The second passage of BMSCs was cultured in the pre-treated supernatant of elastic chondrocytes, which is being referred to as conditioned medium. The conditioned medium was changed every three days. After four weeks of differentiation, BMSCs were digested, suspended and seeded onto glass slides in culture dishes for the chondrogenic differentiation test. In the control group, the BMSCs were cultured in DMEM containing 10\% FBS.

Histological and immunohistochemical staining. After four weeks of culture, the cells were fixed in $4 \%$ (w/v) paraformaldehyde (Sigma-Aldrich) for $15 \mathrm{~min}$ and then subjected to hematoxylin and eosin (Goodbio Technology Co., Ltd., Wuhan, China) and safranin O (Goodbio Technology Co., Ltd.) staining, respectively, to assess cell morphology and chondrocytic differentiation.

Samples were blocked with $3 \% \mathrm{H}_{2} \mathrm{O}_{2}$ and heat was used for antigen retrieval. Antigen retrieval was performed using $0.01 \mathrm{M}$ citrate antigen retrieval solution (Goodbio Technology Co., Ltd.) in a pressure cooker at $100^{\circ} \mathrm{C}$ for $10-15 \mathrm{~min}$. Cells were blocked with $5 \%$ bovine serum albumin (BSA; Sigma-Aldrich) for $30 \mathrm{~min}$ and then incubated at $4^{\circ} \mathrm{C}$ overnight in $0.5 \%$ BSA containing the following antibodies: Type I collagen monoclonal (1:400; ab90395), type II collagen polyclonal (1:50; ab34712), type X collagen monoclonal (1:500; ab49945) and elastin polyclonal (1:500; ab21610) (all Abcam, Cambridge, UK), followed by horseradish peroxidase-conjugated anti-rabbit and anti-rat antibody (Goodbio Technology Co., Ltd.), respectively (1:200 in 0.5\% BSA). Color development was performed with diaminobenzidine tetrahydrochloride (DAB; Maibio, Shanghai, China). Samples were viewed using the Nikon Y-FL 078077 microscope (Nikon, Tokyo, Japan).

Reverse transcription quantitative $(R T-q) P C R$. After chondrogenic induction, total RNA in the experimental and control 
A

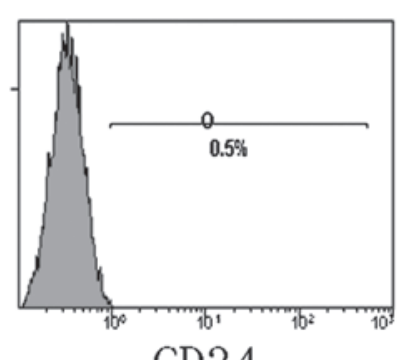

CD34

C

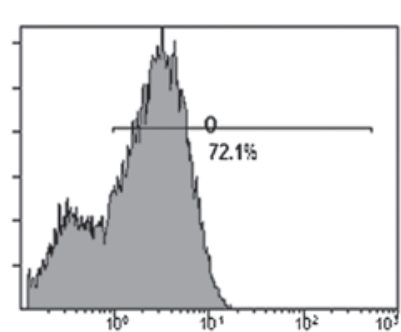

CD29
B

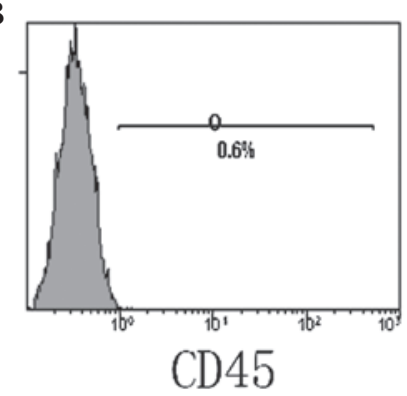

D

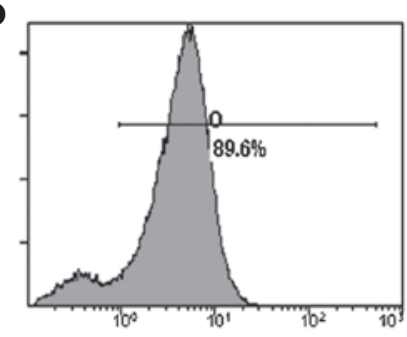

CD90

Figure 1. Flow cytometric analysis of bone marrow-derived stem cells. Cells were negative for mesenchymal stem cell surface markers [(A) CD34 and (B) CD45], while highly expressing the cell-surface markers (C) CD29 and (D) CD90. Graphs are representative of at least three experiments.

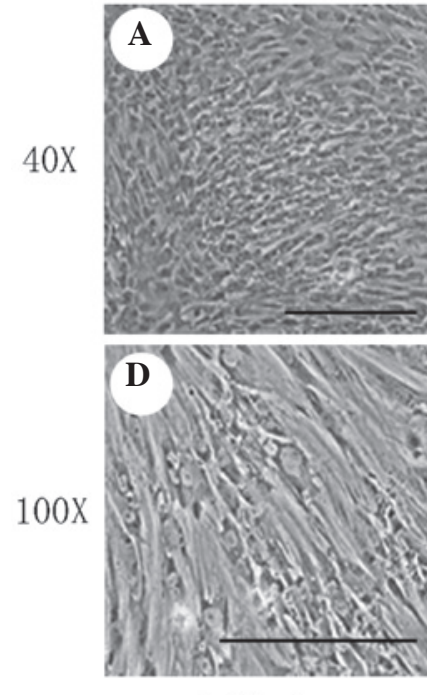

1 Week
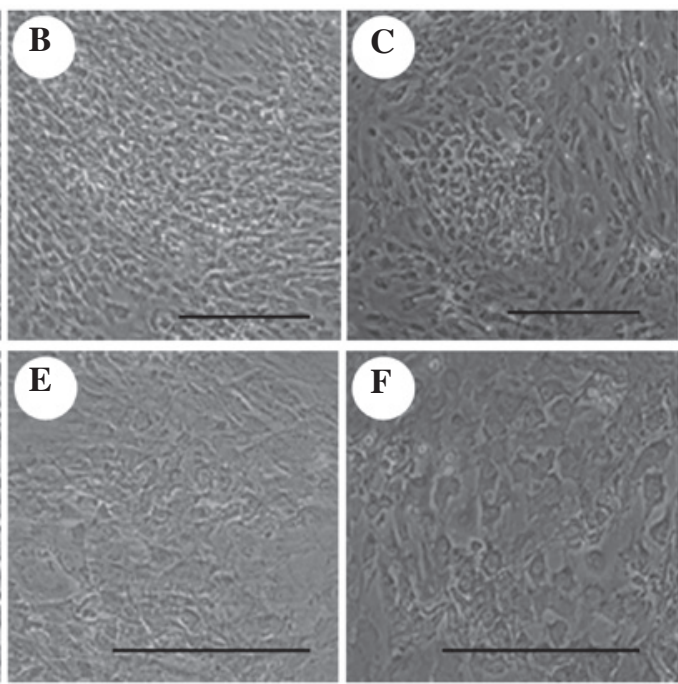

2 Weeks

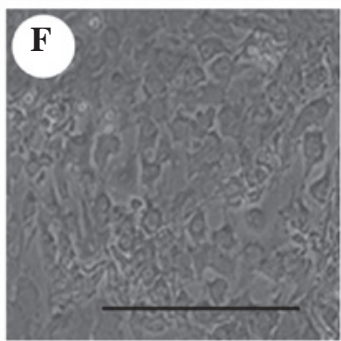

4 Weeks

Figure 2. Cytomorphology. (A and D) After the first week, polygonal cells began to be distinguished, and the cells changed shape from spindle- to cobblestone-like appearance ( $\mathrm{B}$ and $\mathrm{E})$ by the next week and ( $\mathrm{C}$ and $\mathrm{F}$ ) over the next two weeks, which suggested the positive chondro-induction by the chondrocytic supernatant (scale bar, $100 \mu \mathrm{m}$ ).

groups was extracted from each specimen and cDNA was obtained by reverse transcription according to previously described methods (22). PCR was repeated for 40 cycles, and the PCR cycling conditions were as follows: Initial denaturation at $95^{\circ} \mathrm{C}$ for $10 \mathrm{~min}$, annealing at $95^{\circ} \mathrm{C}$ for $15 \mathrm{sec}$, and elongation at $60^{\circ} \mathrm{C}$ for $1 \mathrm{~min}$. The chondrogenic genes (collagen I, collagen II, collagen $\mathrm{X}$ and elastin) were assessed to evaluate chondrogenic differentiation. The primers used in the present study are listed in Table I.

Statistical analysis. Statistical evaluation was performed using Student's $t$ test. Statistical analysis was performed using SPSS version 16.0 (SPSS Inc., Chicago, IL, USA). Results are expressed as the mean \pm standard deviation. $\mathrm{P}<0.05$ was considered to indicate a statistically significant difference between values.

\section{Results}

Flow cytometric analysis. Flow cytometry indicated that the cell-surface markers CD29 and CD90 were highly expressed on BMSCs, while cells were negative for the hematopoietic cell-specific markers CD34 and CD45 (Fig. 1).

Cytomorphology. BMSCs cultured in the supernatant of chondrocytes changed from spindle-shaped into polygonal cells 


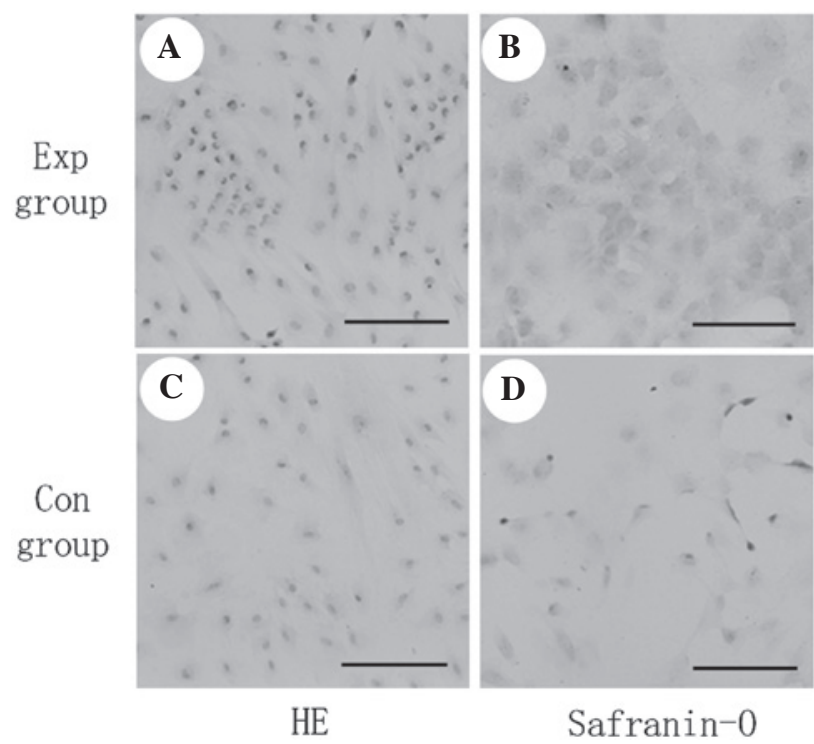

Figure 3. Histological staining. (A and C) At the fourth week, the results of the HE test indicated that BMSCs cultured in the supernatant of elastic chondrocytes shift from a spindle- to cobblestone-like appearance. (D) Compared with the control group of BMSCs, (B) pink staining with Safranin O revealed glycosaminoglycan production by mature chondrocytes (scale bar, $100 \mu \mathrm{m}$ ). Exp, experimental; Con, control; HE, hematoxylin and eosin.

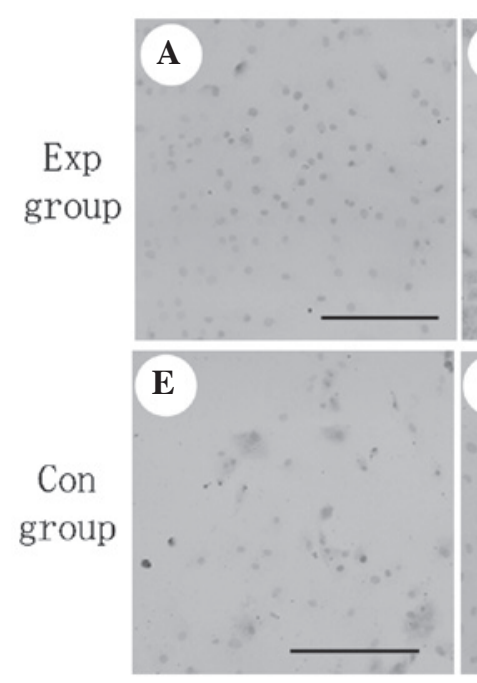

Collagen I
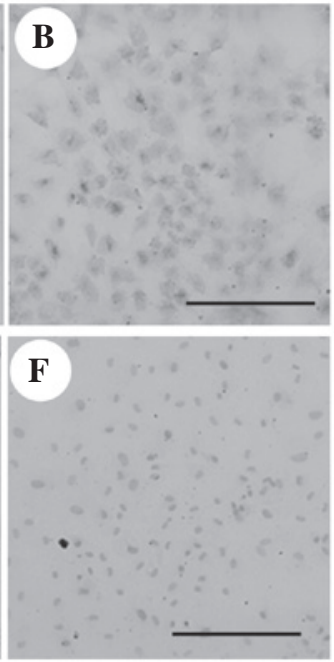

Collagen II
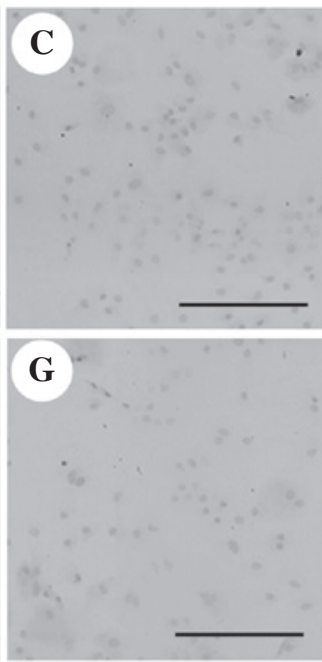

Collagen $X$
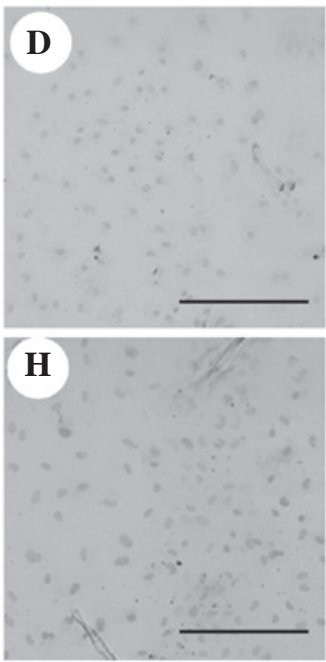

Elast in

Figure 4. Immunohistochemical staining. Positive staining of (B) type II collagen and (D) elastin was observed, whereas the expression of (A) type I and (C) X collagen was inhibited, compared with (E-H) the control group of BMSCs (scale bar, $100 \mu \mathrm{m}$ ). Exp, experimental; Con, control.

with increasing induction time, while BMSCs remained in a spindle- or plate-like shape in the control group (Fig. 2).

In order to investigate the feasibility of chondrogenesis of BMSCs, they were cultured in the supernatant of auricle chondrocytes. At the end of the first week, polygonal cells began to be distinguished, and cells then changed shape from spindle- to cobblestone-like appearance over the next two weeks (Fig. 2), which suggested the positive chondro-induction of the chondrocytic supernatant.

Histological and immunohistochemical staining. Histological and immunohistochemical tests were employed to evaluate the chondrogenesis of BMSCs. The pink staining of safranin $\mathrm{O}$ (Fig. 3) revealed the production of glycosaminoglycan, which was secreted by mature chondrocytes. Furthermore, alongside the

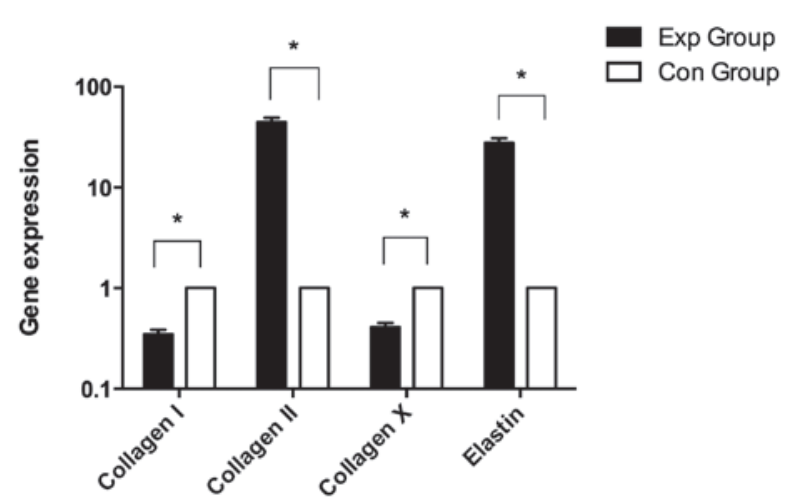

Figure 5. Gene expression analysis. Upregulation of type II collagen and elastin, and downregulation of type I and X collagen were found in the experimental group. Values are expressed as the mean \pm standard deviation, and were normalized to GAPDH. ${ }^{*} \mathrm{P}<0.05$. Exp, experimental; Con, control. 
morphological changes, immunohistochemical analysis showed positive staining for type II collagen and elastin, whereas the expression of type I and X collagen was inhibited (Fig. 4). The above results indicated that the chondrogenic differentiation of BMSCs was improved by the auricle-chondrocyte supernatant, while no hypotrophy was present (Fig. 4). It has been reported that collagen I is an osteogenesis-associated marker and used to assess matrix synthesis during osteoblastic differentiation of BMSCs (23). In addition, collagen $\mathrm{X}$ is an important hypertrophic marker of MSCs (24).

Gene expression. RT-PCR analysis showed significant upregulation of type II collagen and elastin, and significant downregulation of type I and $\mathrm{X}$ collagen $(\mathrm{P}<0.05)$, which was in agreement with the results of the immunohistochemical analysis.

\section{Discussion}

BMSCs, also known as mesenchymal stem cells (MSCs), obtained from the aspiration of bone marrow, are multipotent cells capable of differentiating into cartilage, and are extensively employed in cartilage tissue engineering $(19,22,25)$. The chondrogenesis of BMSCs is a complex and tightly regulated process, and to date, the exact underlying molecular mechanisms have remain to be fully elucidated. It is challenging to create a mimetic chondrogenesis microenvironment through application of TGF- $\beta$ and dexamethone (26). Therefore, in the present study, the supernatant of auricle chondrocytes was used to mimic the chondrogenic differentiation environment in vitro. The objective of the present study was to investigate the chondrogenic differentiation of BMSCs cultivated in the supernatant of auricle chondrocytes. In order to test the properties of BMSCs, flow cytometry was used to detect stem cell surface markers. The BMSCs were then subjected to elastic-chondrocyte differentiation by culturing them in the supernatant of the auricle chondrocytes. Next, histological analysis was performed to evaluate the capacity of elastic-cartilage production. The results showed that the supernatant of chondrocytes had beneficial effects on the chondrogenic differentiation of BMSCs.

The whole-blood adherent method was employed in the present study to obtain BMSCs (27). The surface markers CD29 and CD90 were highly expressed in bone marrow-derived stem cells, while the hematopoietic cell-specific markers CD34 and CD45 were not expressed. A previous study demonstrated that CD90 was associated with the chondrocytic differentiation of MSCs, and it was predominantly expressed in proliferating cells (28). The finding that the surface marker CD90 of BMSCs was highly expressed in the present study was in line with the identification of the capacity of stem cells for mulltilineage differentiation (10). Furthermore, CD90 is regarded to be an important indicator of the chondrogenic differentiation of synovium-derived stem cells (SDSCs) (29). CD29, an integrin sub-unit associated with cell adhesion, is involved in antigen recognition, embryogenesis, hemostasis, tissue restoration and cell migration (27). Apart from that, its expression was shown to indicate the chondrogenic differentiation potency of BMSCs, and to have an important role in the chondrogenesis of SDSCs $(27,28)$.
Direct or indirect chondrocyte-based co-culture of MSCs is thought to be a protocol to generate tissue-engineered cartilage $(15,17,19)$. In the co-culture system, chondrogenic differentiation of MSCs has been shown to require the use of either high-density cell pellet or a scaffold allowing for 3-dimensional culture $(15,30)$. However, Potier et al (31) reported that micro-aggregates had no positive effects on the chondrocytic differentiation of BMSCs. There is currently no consensus regarding the underlying mechanism of the stimulating effect of chondrocytes on MSC-derived chondrogenesis in co-culture systems $(24,32,33)$. Zuo et al (33) suggested that the direct physical contact between chondrocytes and BMSCs has an dominant role, while Fischer et al (17) ascribed the effect to parathyroid hormone-related protein secreted by chondrocytes. The present study utilized a mono-co-culture system without the presence of chondrocytes to provide an improved protocol for the chondrogenic differentiation of BMSCs and to investigate its underlying mechanism. The results of the present study confirmed that the inductive effects of chondroctyes on BMSCs to differentiate was mediated by soluble factors alone, which were present in the supernatant of chondrocytes used for co-culture.

In the present study, it was observed BMSCs began to change their appearance in the first week from the original spindle-like shape to a polygonal shape, and chondrocyte-like cells accumulated with time. Immunohistochemical analysis of type II collagen and glycosaminoglycan (GAG) further confirmed that the supernatant of chondrocytes had an inductive effect on the differentiation of BMSCs, and the chondrocyte-like cells generated from BMSCs showed similar features to those of mature chondrocytes in terms of cartilage-specific collagen production. Collagen type II, the main collagen of the collagen matrix, is the cartilage-specific matrix $(34,35)$. In the present study, type II collagen was present, indicated by an intensive positive staining in the cytoplasm and the pericellular space in the cell sheeting, while staining for type I and X collagen was negative. The ratio of expression of collagen type II-to-I is a well-defined index of chondrocyte differentiation (36). Type I collagen, a marker for de-differentiation and fibration, is the prominent structural element in fibrous cartilage (36-38). Collagen $\mathrm{X}$, an important hypertrophic marker of MSCs, was not present according to immunohistochemical analysis, which suggested that the soluble factors secreted by chondrocytes were able to downregulate the expression of type I and $\mathrm{X}$ collagen and suppress hypertrophy of BMSCs (24). The chondro-induced BMSCs were positive for elastin, an elastic cartilage-specific marker, following the 28-day culture period. The highly elastic mechanical character of elastic cartilage attributes the elasticity of the fiber (39).

In conclusion, the supernatant of elastic cartilage cells was able to induce BMSCs to differentiate into elastic cartilage cells, which may be a promising method for constructing certain sub-types of tissue-engineered cartilage.

\section{Acknowledgements}

The present study was supported by grants from the National Natural Science Foundation of China (grant no. 81272128) and the National 863 program (grant nos. SS2014 and AA020705). The authors appreciate the technical support from Mrs. Lijuan 
Zong, and Mrs. Juanjuan Wu at the Shanghai Key Laboratory of Tissue Engineering (Shanghai 9th People's Hospital, Shanghai Jiao Tong University School of Medicine, Shanghai, China).

\section{References}

1. Hwang CM, Lee BK, Green D, Jeong SY, Khang G, Jackson JD, Atala A, Lee SJ and Yoo JJ: Auricular reconstruction using tissue-engineered alloplastic implants for improved clinical outcomes. Plast Reconstr Surg 133: 360e-369e, 2014.

2. Wachsmuth L, Söder S, Fan Z, Finger F and Aigner T: Immunolocalization of matrix proteins in different human cartilage subtype. Histol Histopathol 21: 477-485, 2006.

3. Suutarla S, Rautio J and Klockars T: The learning curve in microtia surgery. Facial Plast Surg 25: 164-168, 2009.

4. Wilkes GH: Learning to perform ear reconstruction. Facial Plast Surg 25: 158-163, 2009.

5. Bichara DA, O'Sullivan NA, Pomerantseva I, et al: The tissue-engineered auricle: past, present and future. Tissue Eng Part B Rev 18: 51-61, 2012.

6. Reiffel AJ, Kafka C, Hernandez KA, et al: High-fidelity tissue engineering of patient-specific auricles for reconstruction of pediatric microtia and other auricular deformities. PLoS One 8: e56506, 2013

7. Sterodimas A, de Faria J, Correa WE and Pitanguy I: Tissue engineering and auricular reconstruction: a review. J Plast Reconstr Aesthet Surg 62: 447-452, 2009.

8. Nayyer L, Patel KH, Esmaeili A, et al: Tissue engineering: revolution and challenge in auricular cartilage reconstruction. Plast Reconstr Surg 129: 1123-1137, 2012.

9. Patel KH, Nayyer L and Seifalian AM: Chondrogenic potential of bone marrow-derived mesenchymal stem cells on a novel, auricular-shaped, nanocomposite scaffold. J Tissue Eng 4: 2041731413516782, 2013

10. Pittenger MF, Mackay AM, Beck SC, et al: Multilineage potential of adult human mesenchymal stem cells. Science 284 143-147, 1999.

11. Abrahamsson CK, Yang F, Park H, et al: Chondrogenesis and mineralization during in vitro culture of human mesenchymal stem cells on three-dimensional woven scaffolds. Tissue Eng Part A 16: 3709-3718, 2010.

12. Mueller MB and Tuan RS: Functional characterization of hypertrophy in chondrogenesis of human mesenchymal stem cells. Arthritis Rheum 58: 1377-1388, 2008

13. Pelttari K, Winter A, Steck E, et al: Premature induction of hypertrophy during in vitro chondrogenesis of human mesenchymal stem cells correlates with calcification and vascular invasion after ectopic transplantation in SCID mice. Arthritis Rheum 54: 3254-3266, 2006.

14. Qing C, Wei-ding C and Wei-min F: Co-culture of chondrocytes and bone marrow mesenchymal stem cells in vitro enhances the expression of cartilaginous extracellular matrix components. Braz J Med Biol Res 44: 303-310, 2011.

15. Zhang L, He A, Yin Z, et al: Regeneration of human-ear-shaped cartilage by co-culturing human microtia chondrocytes with BMSCs. Biomaterials 35: 4878-4887, 2014.

16. Acharya C, Adesida A, Zajac P, et al: Enhanced chondrocyte proliferation and mesenchymal stromal cells chondrogenesis in coculture pellets mediate improved cartilage formation. J Cell Physiol 227: 88-97, 2012.

17. Fischer J, Dickhut A, Rickert M and Richter W: Human articular chondrocytes secrete parathyroid hormone-related protein and inhibit hypertrophy of mesenchymal stem cells in coculture during chondrogenesis. Arthritis Rheum 62: 2696-2706, 2010.

18. Hwang NS, Varghese S, Puleo C, Zhang Z and Elisseeff J: Morphogenetic signals from chondrocytes promote chondrogenic and osteogenic differentiation of mesenchymal stem cells. J Cell Physiol 212: 281-284, 2007.

19. Xue K, Zhu Y,Zhang Y, Chiang C, Zhou G and Liu K: Xenogeneic chondrocytes promote stable subcutaneous chondrogenesis of bone marrow-derived stromal cells. Int J Mol Med 29: 146-152, 2012 .
20. Friedenstein AJ, Chailakhjan RK and Lalykina KS: The development of fibroblast colonies in monolayer cultures of guinea-pig bone marrow and spleen cells. Cell Tissue Kinet 3: 393-403, 1970.

21. Xu LQ, Huang Y-W, Luo R-Z and Zhang Y-N: Establishment of the retroperitoneal lymph node metastasis model of endometrial VX2 carcinoma in rabbits and observation of its metastatic features. World J Surg Oncol 13: 109, 2015.

22. Xue K, Qi L,Zhou G and Liu K: A two-step method of constructing mature cartilage using bone marrow-derived mesenchymal stem cells. Cells Tissues Organs 197: 484-495, 2013.

23. Zheng B, Jiang J, Luo K, Liu L, Lin M, Chen Y and Yan F: Increased osteogenesis in osteoporotic bone marrow stromal cells by overexpression of leptin. Cell Tissue Res, 2015. (Epub ahead of print)

24. Bian L, Zhai DY, Mauck RL and Burdick JA: Coculture of human mesenchymal stem cells and articular chondrocytes reduces hypertrophy and enhances functional properties of engineered cartilage. Tissue Eng Part A 17: 1137-1145, 2011.

25. He F, Chen X and Pei M: Reconstruction of an in vitro tissue-specific microenvironment to rejuvenate synovium-derived stem cells for cartilage tissue engineering. Tissue Eng Part A 15: 3809-3821, 2009

26. Vinardell T, Thorpe SD, Buckley CT and Kelly DJ: Chondrogenesis and integration of mesenchymal stem cells within an in vitro cartilage defect repair model. Ann Biomed Eng 37: 2556-2565, 2009.

27. Yang YH, Lee AJ and Barabino GA: Coculture-driven mesenchymal stem cell-differentiated articular chondrocyte-like cells support neocartilage development. Stem Cells Transl Med 1: 843-854. 2012

28. Lu T, Huang Y, Wang H, Ma Y and Guan W: Multi-lineage potential research of bone marrow-derived stromal cells (BMSCs) from cattle. Appl Biochem Biotechnol 172: 21-35, 2014.

29. Diaz-Romero J, Gaillard JP, Grogan SP, Nesic D, Trub T and Mainil-Varlet P: Immunophenotypic analysis of human articular chondrocytes: changes in surface markers associated with cell expansion in monolayer culture. J Cell Physiol 202: 731-742, 2005.

30. Albrecht C, Tichy B, Nurnberger S, et al: Gene expression and cell differentiation in matrix-associated chondrocyte transplantation grafts: a comparative study. Osteoarthritis Cartilage 19: 1219-1227, 2011

31. Potier E, Rivron NC, Van Blitterswijk CA and Ito K: Micro-aggregates do not influence bone marrow stromal cell chondrogenesis. J Tissue Eng Regen Med: Apr 2, 2014 (Epub ahead of print)

32. Leppaluoto J, Zeytin F, Ueno N, Ying SY, Ling N and Guillemin R: Myelin basic protein present in the acid extracts of rat hypothalami releases insulin and glucagon from isolated rat pancreatic islets. Acta Physiol Scand 134: 253-261, 1988.

33. Zuo Q, Cui W, Liu F, Wang Q, Chen Z and Fan W: Co-cultivated mesenchymal stem cells support chondrocytic differentiation of articular chondrocytes. Int Orthop 37: 747-752, 2013.

34. Little CJ, Bawolin NK and Chen X: Mechanical properties of natural cartilage and tissue-engineered constructs. Tissue Eng Part B Rev 17: 213-227, 2011.

35. Kuhne M, John T, El-Sayed K, et al: Characterization of auricular chondrocytes and auricular/articular chondrocyte co-cultures in terms of an application in articular cartilage repair. Int J Mol Med 25: 701-708, 2010.

36. Hong E and Reddi AH: Dedifferentiation and redifferentiation of articular chondrocytes from surface and middle zones: changes in microRNAs-221/-222, -140 and $-143 / 145$ expression. Tissue Eng Part A 19: 1015-1022, 2013.

37. Cheng T, Maddox NC, Wong AW, Rahnama R and Kuo AC: Comparison of gene expression patterns in articular cartilage and dedifferentiated articular chondrocytes. J Orthop Res 30: 234-245, 2012

38. von der Mark K, Gauss V, von der Mark H and Müller P: Relationship between cell shape and type of collagen synthesised as chondrocytes lose their cartilage phenotype in culture. Nature 267: 531-532, 1977.

39. Mizuno M, Takebe T, Kobayashi S, et al: Elastic cartilage reconstruction by transplantation of cultured hyaline cartilage-derived chondrocytes. Transplant Proc 46: 1217-1221, 2014. 